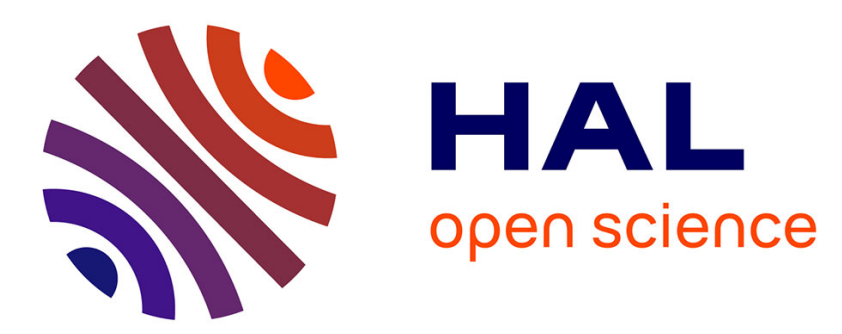

\title{
Ultra-Wide Band Gap in Two-Dimensional Phononic Crystal with Combined Convex and Concave Holes
}

Shan Jiang, Hongping Hu, Vincent Laude

\section{To cite this version:}

Shan Jiang, Hongping Hu, Vincent Laude. Ultra-Wide Band Gap in Two-Dimensional Phononic Crystal with Combined Convex and Concave Holes. physica status solidi (RRL) - Rapid Research Letters, 2018, 12 (2), pp.1700317 (5). hal-02131446

\section{HAL Id: hal-02131446 \\ https://hal.science/hal-02131446}

Submitted on 16 May 2019

HAL is a multi-disciplinary open access archive for the deposit and dissemination of scientific research documents, whether they are published or not. The documents may come from teaching and research institutions in France or abroad, or from public or private research centers.
L'archive ouverte pluridisciplinaire HAL, est destinée au dépôt et à la diffusion de documents scientifiques de niveau recherche, publiés ou non, émanant des établissements d'enseignement et de recherche français ou étrangers, des laboratoires publics ou privés. 


\title{
Ultra-wide band gap in two- dimensional phononic crystal with combined convex and concave holes
}

\author{
Shan Jiang ${ }^{1,2,3}$, Hongping $\mathrm{Hu}^{*}, 1,2,3$, Vincent Laude ${ }^{3}$ \\ ${ }^{1}$ Department of Mechanics, Huazhong University of Science and Technology, Wuhan 430074, P. R. China \\ ${ }^{2}$ Hubei Key Laboratory for Engineering Structural Analysis and Safety Assessment, Huazhong University of Science and Technology, \\ Wuhan 430074, P. R. China \\ ${ }^{3}$ Institut FEMTO-ST, Université de Bourgogne Franche-Comté, CNRS UMR 6174, Besançon 25030, France
}

Received XXXX, revised XXXX, accepted XXXX

Published online XXXX

Key words: Phononic crystal, Ultra-wide band gap, Cross-like holes, L-shaped.

* Corresponding author: e-mail huhp@hust.edu.cn, Phone: +86-27-87543138

A phononic crystal with a ultra-wide band gap is proposed, whose unit cell consists of a cross-like concave hole in the center and four square convex holes at the corners. The dispersion relations, modal kinetic energy ratio and eigenmodes at edges of the band gaps are investigated by using the finite element method. The influence of the geometrical parameters of the convex and concave holes on the band gaps is further analyzed. After optimization, a ultra-wide band gap with gap-to-midgap ratio of $156.0 \%$ is achieved, with the filling fraction keeping a relative small value. Numerical results illustrate that the combination of convex and concave holes is a practicable direction for structural optimization of phononic crystals exhibiting ultra-wide band gaps.

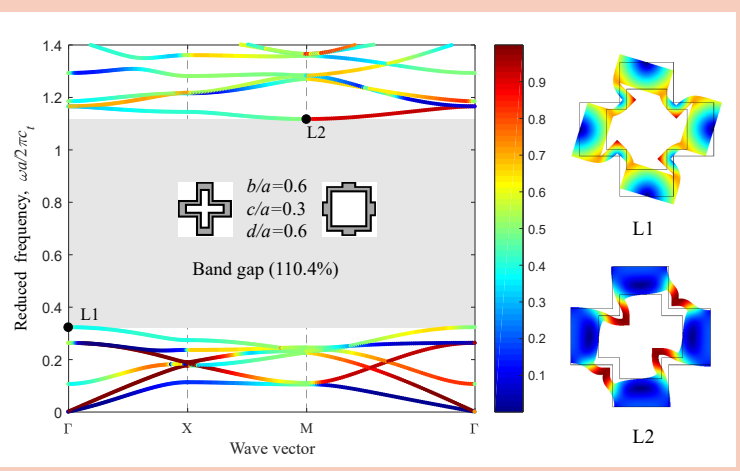

In-plane band structure shown by color of energy ratio and mode shapes for the lower and upper edges of band gap.
1 Introduction Phononic crystals (PnCs) have experienced great developments during the past decades [1]. The most salient trait of PnCs is the existence of band gaps, making them potential candidates for applications in many fields like vibration attenuation, acoustic isolation, sound collimation [2], wave filtering, etc. Previous researches showed that the band gaps depend on several factors, such as material properties of components, lattice structure and its geometrical parameters.

Much interest has been devoted toward PnCs with holes owing to the advantages of simple structure and easy fabrication [3-13]. The introduction of holes into PnCs has been investigated to enhance their performance in many applications, such as waveguide [5-7], light-sound interaction $[8,9]$, and acoustic isolation [10,12]. To obtain broad band gaps, topology optimization is an effective means to explore previously unknown topologies. Many novel and innovative topologies of holey PnCs with broad band gaps have been proposed [12-17]. However, with the genetic algorithms that are applied to solve the optimization problem, a limited population size may not exhaust all optimal-Pareto solutions and corresponding structures. In fact, topology optimization is a systematic way to design PnCs, and the final structure obtained from these algorithms cannot be controlled. The optimized design is highly dependent on the chosen optimization criteria. If additional constraints are not added, the final structure may turn out to be complex and quite difficult to manufacture. 


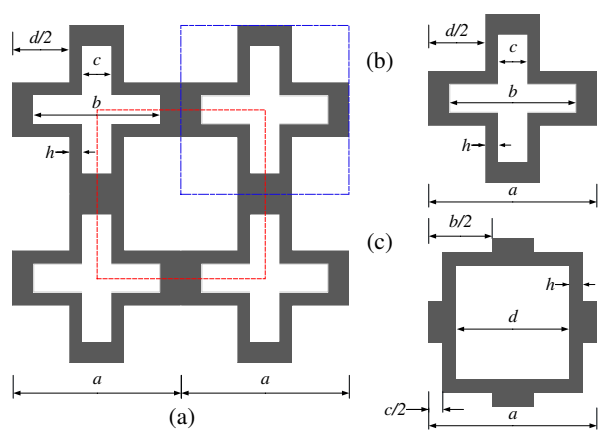

Figure 1 Cross-sections of the proposed 2D square-lattice phononic crystal. (a) An extended unit cell shows the arrangement of concave and convex holes. (b) and (c) show two possible equivalent primitive cells, as outlined by the blue and the red dotted lines in (a). In (b), the primitive cell is centered on the concave cross-like hole surrounded by four square holes placed at the corners. In (c), The primitive cell is centered on the convex square hole surrounded by four L-shaped holes placed at the corners.

For instance, in order to open a wide band gap, a heavy mass and several slim connectors are generally necessary within the unit cell, which leads to large filling fractions of the solid material, in turn bringing about challenges for fabrication, stability of the structure, and overweight. Generally speaking, when comparing two holey PnC designs achieving similar band gap width, the one with the smallest filling fraction is generally preferable.

So far, most of the reported PnC structures, including those obtained from topology optimization, only contain either convex or concave holes. Comparatively, more attention has been paid to PnCs with convex cavities, such as circular or regular polygonal holes. Only a few works have considered their concave counterparts, such as cross-like [18] and snowflake-shaped holes [19], although concaveholey PnCs [18,19] and photonic crystals (PtCs) [20] are both known to generate broad band gaps. Furthermore, except for some topology optimization designs [13,14], the combination of convex and concave holes in the same structure has not been considered explicitly.

Compared to other PnCs that have been discussed in the literature, the PnCs with combined convex and concave holes proposed in this work have the following advantages: (i) ultra-wide band gaps for both in-plane and out-of-plane waves, and a very broad complete band gap; (ii) simple topology with regular holes that can be easily fabricated; (iii) along with a significantly smaller filling fraction of the solid material, much thicker connectors and much lighter masses can induce ultra-wide band gaps that are useful to avoid stability and overweight problems; (iv) ultra-wide band gaps can be obtained in a wide range of geometrical parameters. As a whole, the proposed design is not only beneficial to improve applicability, but also to enhance robustness to manufacturing errors.
Table 1 Comparison on maximum of relative bandwidth (BG\%) between the proposed model and some published structures with structural parameters $\left(h, V_{f}\right)$ for in-plane, out-of-plane, and complete band gaps. BG\% is the ratio of the bandwidth to the mid gap frequency. $h$ and $V_{f}$ denote the thickness of the connector and the filling fraction of solid material.

\begin{tabular}{lllll}
\hline hole & $h / a$ & $\begin{array}{l}\text { In-plane } \\
\mathrm{BG}(\%), V_{f}\end{array}$ & $\begin{array}{l}\text { Out-of-plane } \\
\mathrm{BG}(\%), V_{f}\end{array}$ & $\begin{array}{l}\text { Complete } \\
\mathrm{BG}(\%), V_{f}\end{array}$ \\
\hline$[13]$ & $<1 / 40$ & $131.1,>0.5$ & $135.9,>0.5$ & n.a., n.a. \\
{$[16]$} & $<1 / 40$ & 77.0, n.a. & 122.7, n.a. & 62.6, n.a. \\
{$[17]$} & $1 / 30$ & $121.6,0.80$ & $103.4,0.75$ & $106.8,0.74$ \\
circular & $1 / 40$ & $44.5,0.29$ & $77.5,0.29$ & $44.5,0.29$ \\
“+”[18] & $1 / 40$ & $105.0,0.59$ & $103.2,0.40$ & $86.7,0.59$ \\
"x" [18] & $1 / 40$ & $90.8,0.51$ & $120.8,0.54$ & $81.7,0.54$ \\
the paper & $1 / 40$ & $155.9,0.31$ & $108.8,0.32$ & $108.8,0.32$ \\
the paper & $1 / 30$ & $143.1,0.31$ & $97.6,0.33$ & $97.6,0.33$ \\
\hline
\end{tabular}

2 The model of ultra-wide phononic crystal As shown in Fig. 1, we propose a convex-concave combination 2D square-lattice PnC with a cross-like hole in the center surrounded by four square holes at the corners. Alternatively, the structure can be described as a central square surrounded by $4 \mathrm{~L}$-shaped holes at the corners. In either case, the unit cell is formed by 4 lumps connected by 4 L-shaped connectors. A comparison of relative band gap widths with some published 2D PnC structures is summarized in Table 1. In addition, the filling fraction is given in each case, together with the thickness of the connectors which is the smallest feature limiting the fabrication of the structure. Except for data from references [13,16,17], values are given for the same solid material, Copper, to facilitate comparison. It is worth mentioning that for topology optimization $[13,16,17]$, the optimal designs for in-plane and out-of-plane waves are different structures. Comparing to Ref. [18] with the same thickness of connector $h / a$ of $1 / 30$, the structure has a much smaller filling fraction and a wider in-plane band gap though the out-of-plane and complete band gaps are slightly narrowed. It is seen that the combined concave-convex structure maximizes the combination of large complete band gap and small filling fraction.

As illustrated by Fig. 1, the geometry of the holes is determined by parameters $b, c$ and $d$. All 3 parameters are normalized against the lattice constant $a$ in the calculation. For two-dimensional isotropic materials, wave propagation can be decoupled into in-plane modes and out-of-plane modes. Wave equations for the two modes can be written as

$$
\begin{array}{r}
\mu u_{i, j j}+(\lambda+\mu) u_{j, j i}=-\rho \omega^{2} u_{i} \\
\mu u_{z, i i}=-\rho \omega^{2} u_{z}, \quad i, j=x, y
\end{array}
$$


where $\rho$ and $\omega$ represent mass density and angular frequency, the Lamé constants $\mu$ and $\lambda$ can be expressed by Youngs modulus $E$ and Poisson's ratio $\nu$.

Band structures are computed using the finite element method (FEM). Floquet periodic boundary conditions are imposed on pairs of opposite external boundaries of the unit cell, and free boundary conditions are set along the holes. All solutions are obtained by sweeping wave vector $\mathbf{k}$ along the edges of the irreducible first Brillouin zone. In band structures, reduced frequencies $\left(\omega a / 2 \pi c_{t}\right)$ are presented as a function of the reduced wavenumber $(\mathrm{ka} / 2 \pi)$, with $c_{t}(=\sqrt{\mu / \rho})$ denoting the transverse shear wave velocity. The modal kinetic energy ratio $e_{r}$ in the direction of propagation is defined as

$$
e_{r}=\int_{A} u_{\|}^{2} d A / \int_{A} u^{2} d A
$$

where $u$ and $u_{\|}$stand for the displacement and its component along the direction of propagation, and $A$ denotes the area of the unit cell.

3 Numerical results and discussions In the following discussion, all PnCs are made of Copper, with mass density $\rho=8900 \mathrm{~kg} / \mathrm{m}^{3}$, Young's modulus $E=120$ GPa, and Poisson's ratio $\nu=0.3$. Figs. 2(a) and 2(b) display the band structures for in-plane mode and for out-ofplane mode, respectively. The geometrical parameters are $b / a=0.6, c / a=0.3, d / a=0.6$, and the thickness of the connectors is consequently $h=(a-c-d) / 2=a / 20$. The color of bands represents the modal kinetic energy ratio $e_{r}$. For the in-plane mode, a ultra-wide band gap $(\mathrm{BG} \% \geq 100 \%)$ spans from 0.322 to 1.116 with a BG\% of $110.4 \%$. For the out-of-plane mode, a band gap between 0.436 and 1.003 is achieved with a BG\% of $78.7 \%$. The band gap for out-of-plane mode also is a complete band gap, as it is fully contained in the in-plane band gap.

In order to understand the mechanism of band gap formation, 4 eigenmodes selected at the edge frequencies of the band gaps are presented in Fig. 2(c). The topology can be considered to be composed of 4 lumps and $4 \mathrm{~L}$-shaped narrow connectors. For the 4 eigenmodes, one can consider that the lumps vibrate mostly as rigid bodies since they withstand much less deformations than the thin L-shaped connectors. For in-plane waves, the lower edge mode L1 is dominated by rotatory motion of the 4 lumps around their centroids and flexural vibrations of the 4 L-shaped connectors. The lump-connector system acts as a mass-spring system with a low resonance frequency. The upper edge mode L2 corresponds to the resonance of the connectors while the lumps remain almost stationary. Its two ends being fixed, every connector vibrates locally with coupled extension and flexure, and hence has a high eigenfrequency. Furthermore, two connectors move with the polarization in the propagation direction, while the other two connectors vibrate perpendicularly to the propagation direction. Similarly, the lower and upper edge modes S1 and S2 are
Table 2 Maximums of BG\% and corresponding optimal geometrical parameters for different connector thicknesses $h$.

\begin{tabular}{lllll}
\hline & \multicolumn{2}{c}{ In-plane } & \multicolumn{2}{c}{ Out-of-plane } \\
$h / a$ & $\mathrm{BG}(\%)$ & $b / a, c / a, d / a$ & $\mathrm{BG}(\%)$ & $b / a, c / a, d / a$ \\
\hline 0.065 & 102.6 & $0.72,0.26,0.61$ & 65.8 & $0.58,0.30,0.57$ \\
0.060 & 107.7 & $0.72,0.28,0.60$ & 69.8 & $0.58,0.28,0.58$ \\
0.055 & 113.4 & $0.70,0.28,0.61$ & 74.0 & $0.60,0.30,0.59$ \\
0.050 & 119.3 & $0.70,0.30,0.60$ & 78.7 & $0.60,0.30,0.60$ \\
0.045 & 125.8 & $0.68,0.30,0.61$ & 83.6 & $0.60,0.32,0.59$ \\
0.040 & 132.5 & $0.68,0.30,0.62$ & 89.6 & $0.60,0.32,0.60$ \\
0.035 & 140.0 & $0.66,0.32,0.61$ & 94.8 & $0.62,0.32,0.61$ \\
0.030 & 147.8 & $0.66,0.34,0.60$ & 101.8 & $0.62,0.32,0.62$ \\
0.025 & 155.9 & $0.64,0.34,0.61$ & 108.8 & $0.62,0.34,0.61$ \\
\hline
\end{tabular}

antisymmetrical and symmetrical out-of-plane modes, respectively. A similar mechanism was analyzed for PnCs with several topologies [15], and with cross-like holes [18]. Owing to combination of convex holes with concave holes, the connectors have a L-shaped structure. As a result, the connectors have a small equivalent bending stiffness and a large equivalent tensile stiffness. The eigenfrequency of the lower edge then becomes smaller even though the lump does not have a large effective mass, while the eigenfrequency of the upper edge becomes larger. Compared with the 2D PnCs listed in Table 1 and with a 3D PnC [12] with a same thickness of the connectors, the proposed PnC opens an ultra-wide band gap while at the same time keeping a significantly smaller filling fraction.

As a note, at the edge modes L1 and L2, the modal kinetic energy ratio $e_{r}=0.5$, which is in agreement with the previous discussion of vibration modes. $e_{r}=0.5$ means that the kinetic energy of the longitudinal displacement is equal to the kinetic energy of the transverse displacement. Hence, we call this condition energy balance. We further find that the energy balance phenomenon also appears in the corresponding edge modes of the circularholey or cross-holey PnC.

To obtain the largest band gaps, optimization of geometrical parameters is conducted inductively. Firstly, the influence of the parameters $b$ and $c$ of the concave hole on band gap widths are presented in Figs. 3(a) and 3(b) for in-plane modes and out-of-plane modes respectively. The thickness of the connectors is $h=a / 20$ as set by $(c+d) / a=0.9$. Upon observing Fig. 3, ultra-wide band gaps are available in a large sizes range of the concave hole. This feature is not only beneficial to improve applicability, but also to enhance robustness to manufacturing errors. A maximum in-plane BG\% of $119.3 \%$ is obtained by optimal geometrical parameters $b / a=0.7$ and $c / a=0.3$. The maximum out-of-plane BG\% is $78.7 \%$ by $b / a=0.6$ and $c / a=0.3$. Owing to the combined action of concave hole parameters $b$ and $c$, and convex hole parameter $d$ as $V_{f}=1-\left(d^{2}+2 b c-c^{2}\right) / a^{2}$, these geometrical parame- 

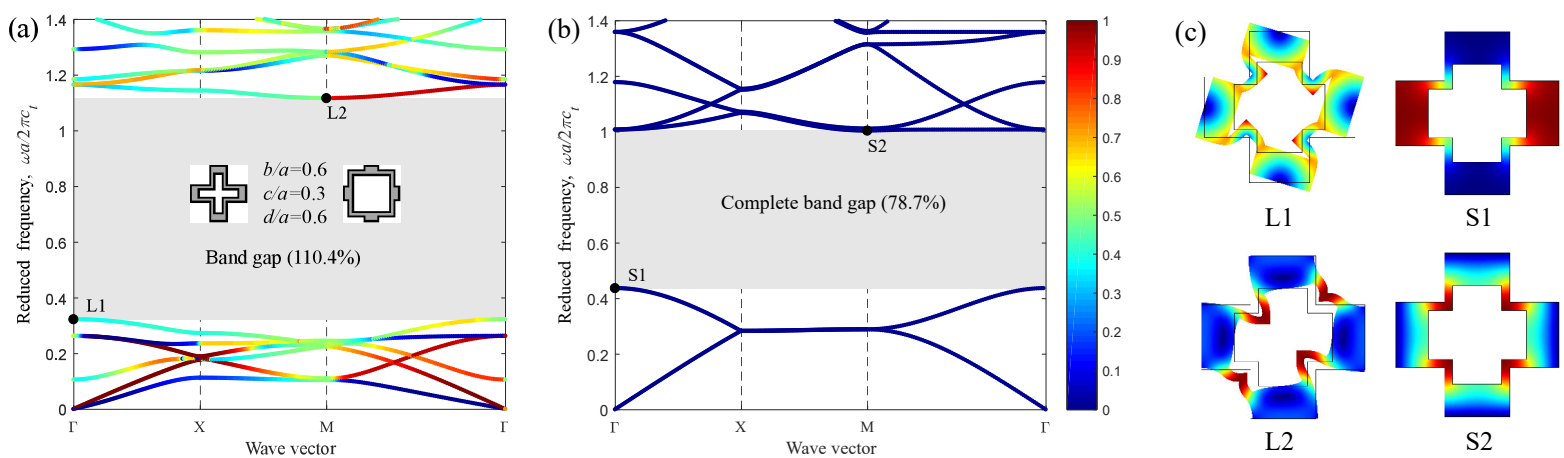

Figure 2 Band structures of (a) in-plane mode and (b) out-of-plane mode for the proposed PnC, and (c) mode shapes for the lower and upper edges of band gaps. Color bar represents the modal energy ratio $e_{r}$ in the propagation direction.
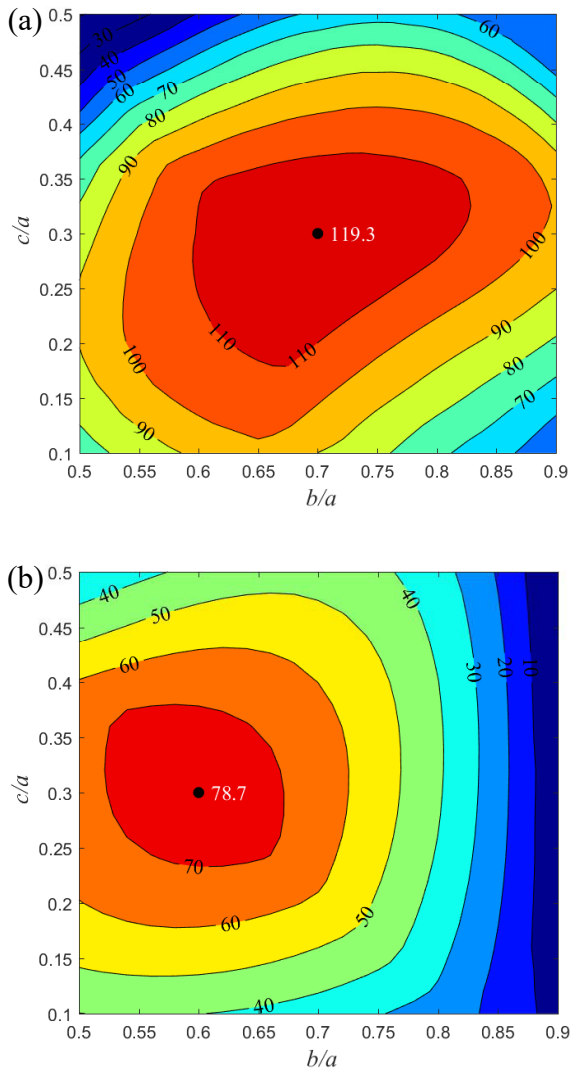

Figure 3 Band gap width of (a) In-plane mode and (b) out-of-plane mode as a function of geometrical parameters $b / a$ and $c / a$. The connector thickness is $a / 20$ as set by $(c+d) / a=0.9$.

ters correspond to small filling fractions 0.31 and 0.39 . It is quite different from other either concave or convex holey ultra-wide PnCs $[15,18]$, for which the maximum BG\% always appears when the filling fraction is large.
The optimum band gap widths and the corresponding optimal geometrical parameters are presented as a function of the thickness $h$ of the connectors in Table 2. BG\% increases from $97.9 \%$ to $155.9 \%$ for in-plane modes, and from $61.7 \%$ to $108.8 \%$ for out-of-plane modes, as $h$ decreases from $0.065 a$ to $0.025 a$. Fig. 4 illustrates edge frequencies and band gap widths vary versus the normalized side length $d / a$ of convex holes, for fixed $b / a=$ 0.6 and $c / a=0.3$. In-plane and out-of-plane band gaps are represented by blue and magenta regions respectively. Their overlap defines the complete band gaps. The lower edges of both in-plane and out-of-plane band gaps decrease monotonously with $d / a$. The upper edge of the out-ofplane band gap increases monotonously with $d / a$, but the upper edge of the in-plane band gap has much less variations. Globally, the in-plane and out-of-plane band gap widths both increase monotonically. As the red dash line shows, the in-plane band gap width reaches a maximum of $156.0 \%$ for $d / a=0.65$.

The bending stiffness $\kappa$ of the L-shaped connectors is proportional to the cubic power of the thickness $h$. As $d / a$ increases, the connector thickness decreases due to the relation $h=(a-c-d) / 2$. The mass of the lumps $m=\rho(a-d)(a-b) / 2$ also decreases. Therefore, the lower edge of the in-plane band gap determined roughly by $\sqrt{\kappa / m}$ still decreases monotonically. In contrast, for its upper edge, the connectors with two ends fixed vibrate mainly according to an extension mode whose eigenfrequency is independent of the thickness. As a result, its upper edge frequency varies only a little. As antisymmetrical and symmetrical modes respectively, the edge frequencies of the out-of-plane band gap S1 and S2 have opposite tendencies with the variation of $d / a$.

4 Conclusions An ultra-wide 2D square-lattice PnC has been proposed that possesses ultra-wide band gaps for a large range of geometrical parameters, while keeping thick connectors and a relatively small filling fraction. As combination of convex and concave holes, the struc- 


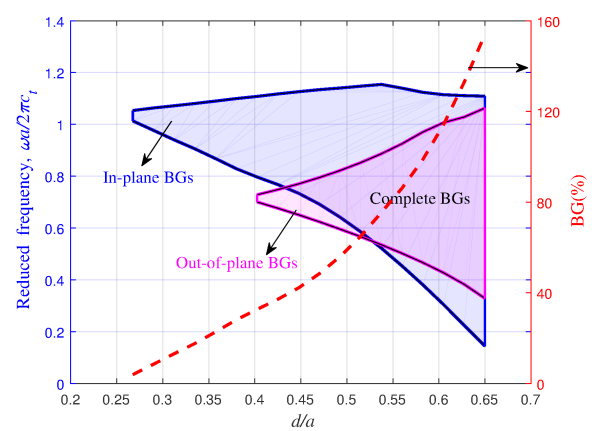

Figure 4 Variation of edge frequencies and band gap widths with geometrical parameter $d / a$, for fixed $b / a=$ 0.6 and $c / a=0.3$. The thickness of connectors $h / a$ changes from 0.225 to 0.025 accordingly. The red dash line shows the variation of the band gap width $\mathrm{BG} \%$ for in-plane modes.

ture of L-shaped connectors and lumps is critical to induce ultra-wide band gaps. A maximum band gap width of up to $156.0 \%$ was found after optimization. Structures combining convex and concave holes would further be good starting designs for further topology optimization of phononic crystals where the filling fraction has to be minimized as the band gaps are maximized.

Acknowledgements This work was supported by the National Natural Science Foundation of China (11272126, 51435006, and 51421062) the Fundamental Research Funds for the Central Universities, HUST: Nos. 2016JCTD114 and 2015TS121. Hu and Jiang also acknowledge the support from China Scholarship Council (Grants Nos. 201606165026 and 201606160045).

\section{References}

[1] V. Laude, Phononic Crystals: Artificial Crystals for Sonic, Acoustic, and Elastic Waves (Walter de Gruyter GmbH, 2015).

[2] J. Shi, S. S. Lin and T. J. Huang Appl. Phys. Lett. 923152 (2008).

[3] S. Jiang, H. Chen, L. X. Dai, H. P. Hu and V. Laude, Compos. Struct. 176, 294-303 (2017).

[4] Y. Liu, J. Y. Su, Y. L. Xu, L. Ya and X. C. Zhang, Ultrasonics 49, 276-80 (2017).

[5] F. C. Hsu, C. I. Chiung, J. C. Hus, T. C. Huang, C. H. Wang and P. Chang, Appl. Phys. Lett. 96, 051902 (2010).

[6] C. S. Li, D. Huang, J. R. Guo and J. J. Nie, Phys. Lett. A 38 , 2633-37 (2013).

[7] E. Coffy, T. Lavergne, M. Addouche, S. Euphrasie, P. Vairac and A. Khelif, J. Appl. Phys. 118, 214902 (2015).

[8] F. L. Hsiao , C. Y. Hsieh , H. Y. Hsieh and C. C. Chiu, Appl. Phys. Lett. 100, 171103 (2012)

[9] Y. Pennec, V. Laude, N. Papanikolaou, B. Djafari-Rouhani, M. Oudich, S. E. Jallal, J. C. Beugnot, J. M. Escalante and A. Martinez, Nanophotonics 3, 413-40 (2014)

[10] D. Yudistira, Y. Pennec, R. B. Djafari, S. Dupont and V. Laude, Appl. Phys. Lett. 100, 061912 (2012).
[11] D. Mousanezhad, S. Babaee, R. Ghosh, E. Mahdi, K. Bertoldi and A. Vaziri, Phys. Rev. B 92, 104304 (2015).

[12] L. D'Alessandro, E. Belloni, R. Ardito, A. Corigliano and F. Braghin, Appl. Phys. Lett. 109, 221907 (2016).

[13] Y. F. Li, X. D. Wang and S. W. Zhou, Materials. 9, 186 (2016).

[14] S. Hedayatrasa, K. Abhary, M. Uddin and C. T. Ng, J. Mech. Phys. Solids 89, 31-58 (2016).

[15] H. W. Dong, Y. S. Wang and C. Z. Zhang, IEEE Photonics J. 9, 1-16 (2017).

[16] O. R. Bilal and M. I. Hussein, Phys. Rev. E 84, 065701 (2011).

[17] H. W. Dong, X. X. Su and Y. S.Wang, J. Phys. D: Appl. Phys. 47, 155301 (2014).

[18] Y. F. Wang, Y. S. Wang and X. X Su, J. Appl. Phys. 110, 113520 (2011).

[19] A. H. Safavi-Naeini, J. T. Hill, S. Meenehan, J. Chan S. Gröblacher and O. Painter, Phys. Rev. Lett. 112, 153603 (2014).

[20] T. Kim, C. Seo. IEEE Microwave Guided Wave Lett. 10, 13-5 (2000) 